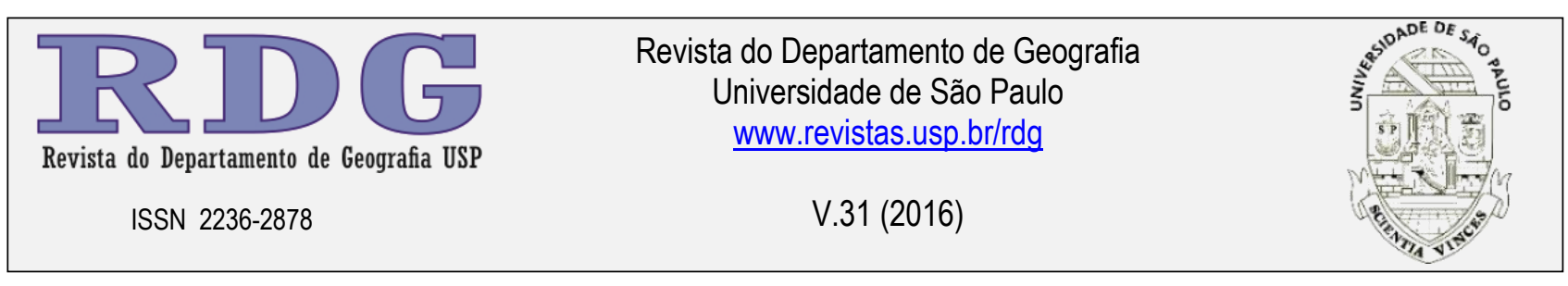

\title{
TENDÊNCIA DO CLIMA DO SEMIÁRIDO FRENTE AS PERSPECTIVAS DAS MUDANÇAS CLIMÁTICAS GLOBAIS; O CASO DE ARARIPINA, PERNAMBUCO
}

\author{
SEMIARID CLIMATE TREND IN GLOBAL CLIMATE CHANGE PERSPECTIVES; \\ THE CASE OF ARARIPINA IN PERNAMBUCO
}

Francinete Francis Lacerda

Instituto Agronômico de Pernambuco francis.lacerda@ipa.br

Paulo Nobre

Instituto Nacional de Pesquisas Espaciais

Maria Do Carmo Martins Sobral

Universidade Federal de Pernambuco

Geraldo Majella Bezerra Lopes

Instituto Agronômico de Pernambuco

Eduardo Delgado Assad

Empresa Brasileira de Pesquisa Agropecuária

Recebido (Received): 27/04/2016 Aceito (Accepted): 22/06/2016 DOI: http://dx.doi.org/10.11606/rdg.v31i0.114843

\begin{abstract}
Resumo: Este artigo mostra os resultados de pesquisa para o semiárido pernambucano utilizando séries temporais diárias de temperatura e de precipitação com mais de 40 anos, com cenários futuros de mudanças do clima para o período de 2010 a 2050. Para determinar as tendências de mudanças do clima, presente e futuro, foram utilizadas tendências de mudanças climáticas, resultados de balanço hídrico e saídas do modelo atmosférico regional aninhado ETA, em cenário global do modelo climático aninhado em dois modelos climáticos globais, HadCM3 e BESM. Os resultados indicaram tendência de aumento das temperaturas máximas, redução das temperaturas mínimas, redução da pluviosidade, média anual, indicando tendência à aridização na região.
\end{abstract}

Palavras-chave: cenários climáticos; aridização; extremos de temperatura.

\begin{abstract}
This article shows results of a research carried out for the semiarid of Pernambuco, Brazil using daily time series of temperature and precipitation over 40 years, with future scenarios of climate change for the period 2010-2050. To determine present and future climate change trends, water balance results and outputs of the regional atmospheric model ETA nestled in global scenario of climate model nested in two global climate models HadCM3 and BESM were used. The results showed increased trend in maximum temperature, reducing minimum temperatures, reduced average annual rainfall, indicating tendency to aridization in the region.
\end{abstract}

Keywords: climate change scenarios, aridization, temperature extremes 


\section{INTRODUÇÃO}

Estudos recentes, no Brasil e no mundo, sobre o aquecimento global e segurança alimentar indicam que a mudança climática pode afetar a produção de alimentos e fazer com que áreas de risco aumentem (IPCC, 2013; ASSAD e PINTO, 2008). Em se confirmando o aumento das temperaturas, o atual zoneamento agrícola brasileiro deverá ser modificado para contemplar a nova realidade do clima (NOBRE e ASSAD, 2005).

No caso de Pernambuco, evidências de alterações climáticas já foram encontradas, mostrando que o aumento de temperatura do ar é crescente (LACERDA et al., 2010). O Modelo Brasileiro do Sistema Terrestre (BESM), indica um quadro de modificação no comportamento das Temperaturas da Superfície do Mar (TSM) sobre o Atlântico Tropical num cenário de aumento de $\mathrm{CO} 2$ nas próximas décadas, com o aumento da ocorrência de anos de seca, alternados com anos de precipitação abundante sobre o Nordeste do Brasil (NOBRE et al., 2013).

A região Nordeste do Brasil, em geral e o estado de Pernambuco, em especial, estão vulneráveis aos processos de desertificação, à ocorrência de eventos extremos do clima tais como secas severas e chuvas intensas. Segundo o Relatório do Painel Brasileiro de Mudanças Climáticas (PBMC, 2013), é provável que o semiárido nordestino tenha sua precipitação reduzida em até $20 \%$ até 2040 , com aumento de temperatura de até $1^{\circ} \mathrm{C}$. Este relatório, também, indica que todo o Brasil deverá ficar ao menos $3^{\circ} \mathrm{C}$ mais quente até o fim do século; as precipitações aumentariam em $30 \%$ nas regiões Sul e Sudeste e diminuiriam em até $40 \%$ nas regiões Norte e Nordeste. Pernambuco tem $70 \%$ de sua área no semiárido e nessa região a precipitação acumulada, em média, é inferior a 700 $\mathrm{mm} /$ ano. No setor oeste dessa região está a microrregião do Araripe, onde o principal período chuvoso ocorre entre os meses de janeiro a abril.

Estudos de detecção de mudanças climáticas em Pernambuco (LACERDA et al., 2015), revelaram um aumento de $4^{\circ} \mathrm{C}$ na temperatura máxima diária no período de 1961 a 2009 e diminuição da precipitação em $275 \mathrm{~mm}$ (correspondendo a 57\% do total anual) (Nobre, 2011). Nota-se que a diminuição dos totais pluviométricos anuais é observada globalmente nas regiões tropicais entre $10^{\circ} \mathrm{S}$ e $10^{\circ} \mathrm{N}$, assim como o aumento da frequência da ocorrência de precipitações episódicas intensas associadas às mudanças climáticas globais (TRENBERTH et al., 2007; MARENGO et al., 2007).

Com base nesse contexto o objetivo com esse trabalho foi o de identificar as tendências de temperatura e precipitação sobre Araripina (PE/Brasil) utilizando séries temporais, realizar o balanço hídrico e formular cenários e tendências climáticas para o município utilizando um modelo atmosférico regional.

\section{MATERIAIS E MÉTODOS}

Foram utilizadas séries históricas com 50 anos de dados de precipitação e de temperatura diária do ar no período que abrangeu as décadas de 1960 a 2010 para a localidade de Araripina em Pernambuco (Lat $7^{\circ} 34^{\prime} 34^{\prime \prime S}$; Long 40²9'54"O). O Software utilizado para o processamento e controle de qualidade dos dados (verificação de dados espúrios e falhas nas séries históricas de chuva e temperatura) foi o RClimdex (ZHANG E YANG, 2004), também, utilizado para o cálculo das tendências nas séries históricas diárias de temperatura e precipitação. Os metadados da estação de Araripina (localização, posicionamento e qualidade de funcionamento dos equipamentos etc.) foram averiguados com intuito de garantir fidedignidade dos dados originais e corrigidos manualmente, quando necessário, no banco de dados.

Para verificar a qualidade das séries históricas diárias e realizar o cálculo das tendências de precipitação e temperatura, foi utilizada metodologia de Zhang et al. (2005) e Haylock et al. (2006), também, usada por diversos outros autores (SANTOS e BRITO, 2007; SILVA e AZEVEDO, 2008; IPCC, 2007; SILLMANN et al., 2013a; e SILLMANN et al., 2013b). 
Os seguintes cálculos estatísticos foram realizados com o RCLIMDEX: tendência linear calculada pelo método dos mínimos quadrados, nível de significância estatística da tendência (valor p), coeficiente de determinação (r2) e erro padrão da estimativa. Foram consideradas como tendências climáticas os índices que apresentaram tendência linear (negativa ou positiva), superior ao erro padrão da estimativa e estatisticamente significativos (valor de $\mathrm{p}<0,1$ ), obtidos pelo teste $\mathrm{t}$ de student a saber: 99\% para $\mathrm{p}<0,01,95 \%$ para $\mathrm{p}<0,05,90 \%$ para $\mathrm{p}>0,05$ e $\mathrm{p}<0,1$ e sem significância $\mathrm{p}>0,1$.

O segundo passo foi o de calcular os balanços hídricos anuais (MCCABE e MARKSTROM, 2007) para Araripina, considerando os dados diários de precipitação e temperatura do ar em 40 anos das observações meteorológicas, de 1960 a 2010. Os balanços hídricos foram calculados para uma capacidade de água disponível (CAD) de $100 \mathrm{~mm}$. As variáveis de saídas mensais analisadas, do balanço hídrico, foram Evapotranspiração Potencial (ETP) e o Armazenamento de água no solo (ARM).

O terceiro passo consistiu na realização de simulações numéricas para o clima atual e o futuro, fazendo uso do modelo regional Eta atmosférico (CHOU et al., 2011) aninhado em dois modelos climáticos globais, HadCM3 (CHOU et al., 2005; AMBRIZZI et al., 2007) e BESM (NOBRE et al., 2013). As variáveis prognósticas foram: temperatura, umidade, vento horizontal, pressão à superfície, energia cinética turbulenta, umidade e temperatura do solo e hidrometeoros das nuvens. O modelo possui esquema de microfísica de nuvens. O domínio do modelo abrange a maior parte da América do Sul. A temperatura da superfície do mar é atualizada a cada dia da integração. Para utilizar os dados das simulações do modelo climático global HadCM3 com o objetivo de fazer a regionalização ("downscaling") para o domínio específico com o modelo Eta/CPTEC, foi necessária a preparação da "interface" e adaptar os dados do Hadley Center de maneira adequada para a utilização destes como dados de entrada no modelo Eta/CPTEC. O modelo Eta/CPTEC utilizou dados de entrada em formato GRIB em níveis isobáricos (padrão de reanálise e padrão do modelo americano AVN). Os dados foram vento zonal e meridional, altura da superfície isobárica, pressão à superfície e umidade específica.

Os cenários regionais foram gerados para a região Nordeste, com uma resolução espacial de $40 \mathrm{~km}$ ao longo de todo o oceano Atlântico tropical e a porção tropical da América do Sul. Os experimentos com o modelo Eta foram utilizados para duas concentrações de $\mathrm{CO} 2$ atmosférico: 380 ppmv (1960 a 2000) e 760 ppmv (2010-2050). As simulações para o clima atual (1960-2000) e cenários futuros (2010-2050) foram feitas usando condições de contorno do modelo climático global HadCM3, como utilizadas em estudos anteriores (CHOU et al., 2005; AMBRIZZI et al., 2007).

As simulações com o modelo brasileiro (BESM), um modelo global-oceano-atmosferabiosfera-criosfera acoplado (NOBRE et al., 2013), foram utilizadas para caracterizar o clima presente. Para efeito comparativo das simulações foram utilizados os dados do NCEP/NCAR Reanalysis 1 (NOAA, 2015).

\section{RESULTADOS E DISCUSSÕES}

Em Araripina, constatou-se a tendência de aumento das temperaturas máximas, diminuição das máximas temperaturas mínimas e diminuição da pluviosidade anual. Esses resultados estão expressos na Tabela 1. Para as análises de tendências aplicadas às séries históricas de precipitação e de temperatura foram considerados os valores correspondentes às máximas das temperaturas máximas, mínimas das temperaturas máximas, bem como, as máximas e mínimas das temperaturas mínimas. Em Araripina, as tendências positivas corresponderam às temperaturas máximas. Essas tendências apresentaram valores de $0,72 \mathrm{oC}$ para as máximas das temperaturas máximas e 1,82oC para as mínimas das temperaturas máximas, por década. A tendência negativa foi de $0,40 \mathrm{oC}$ por década, para as máximas das temperaturas mínimas e positiva para as mínimas das temperaturas mínimas $(0,05$ oC por década). Já as tendências das mínimas das temperaturas mínimas 
apresentaram baixa significância estatística. As análises de tendência da pluviosidade evidenciaram valores negativos de 2,54 mm/ano (Tabela 1).

Tabela 1- Tendências (T) (em ${ }^{\circ} \mathrm{C} /$ década) para os extremos de temperaturas máximas e mínimas e de precipitação pluviométrica, em Araripina (mm/ano).

\begin{tabular}{c|c|c|c|c|c|c|c|c|c}
\hline \multicolumn{2}{c|}{ Temperatura máxima } & \multicolumn{2}{c|}{ Temperatura mínima } & \multicolumn{2}{c}{ Precipitação } \\
\hline \multicolumn{2}{c|}{$\begin{array}{c}\text { Máxima das } \\
\text { máximas }\end{array}$} & \multicolumn{2}{c|}{$\begin{array}{c}\text { Mínima das } \\
\text { máximas }\end{array}$} & \multicolumn{2}{c|}{$\begin{array}{c}\text { Máxima das } \\
\text { mínimas }\end{array}$} & \multicolumn{2}{c}{$\begin{array}{c}\text { Mínima das } \\
\text { mínimas }\end{array}$} & \multicolumn{2}{c}{ mm } \\
\hline \multirow{2}{*}{ T } & $\begin{array}{c}\text { Valor } \\
\text { médio }\end{array}$ & $\mathrm{T}$ & $\begin{array}{c}\text { Valor } \\
\text { médio }\end{array}$ & $\mathrm{T}$ & $\begin{array}{c}\text { Valor } \\
\text { médio }\end{array}$ & $\mathrm{T}$ & $\begin{array}{c}\text { Valor } \\
\text { médio }\end{array}$ & $\mathrm{T}$ & $\begin{array}{c}\text { Valor } \\
\text { médio }\end{array}$ \\
\hline $0,72(*)$ & 35,1 & $1,82(*)$ & 24,9 & $-0,40$ & 21,4 & $0,05(*)$ & 13,2 & $-2,54$ & 794 \\
\hline
\end{tabular}

(*) significância estatística para os índices de tendência; em negrito 99\%, em preto $90 \%$

A taxa de aumento da temperatura máxima foi de 1,28 oC/década, no período estudado. $\mathrm{O}$ resultado indicou que as temperaturas mínimas estão diminuindo a uma taxa de 0,09 oC/década (Figura 1).

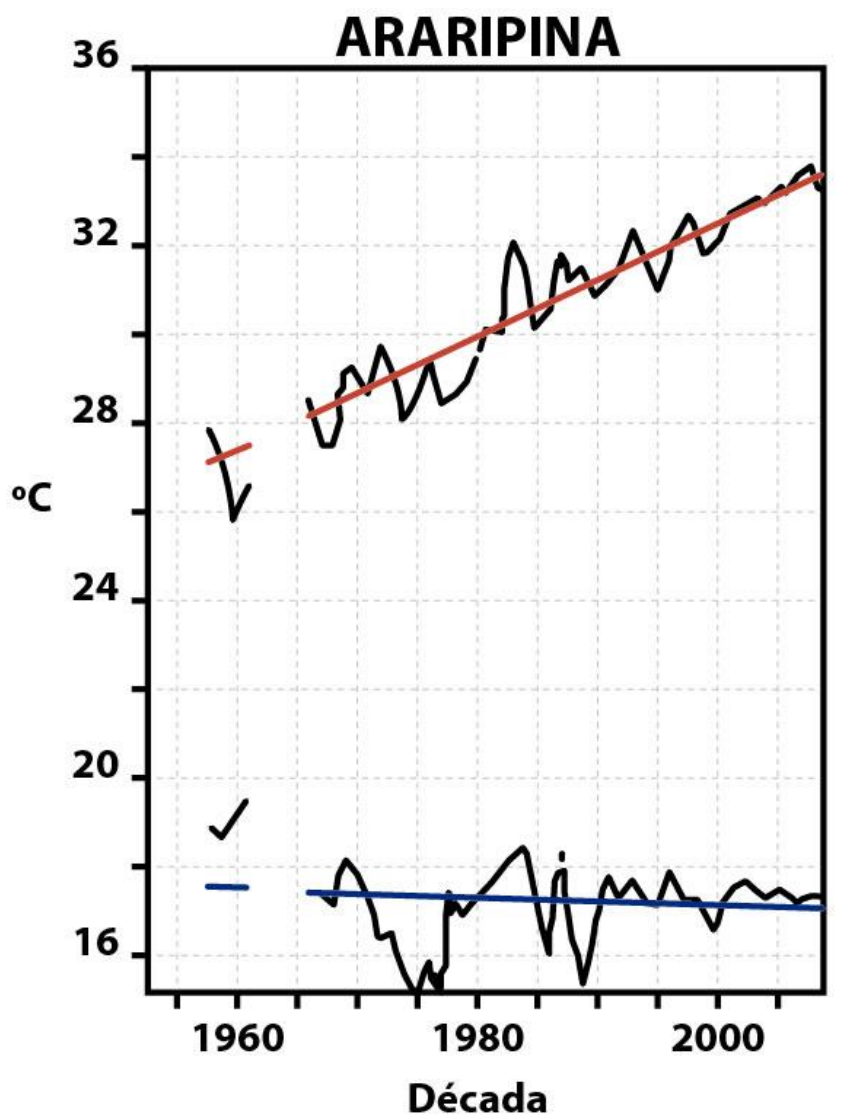

Figura 1: Tendências das temperaturas diárias $\left({ }^{\circ} \mathrm{C} /\right.$ década) máximas e mínimas para Araripina. A linha vermelha indica a tendência das temperaturas máximas e a azul a tendência das temperaturas mínimas, para os períodos de 1960 a 2010. 
A taxa de variação decadal (diferença mensal entre a tendência de aumento da temperatura máxima e a tendência da temperatura mínima, por década) da temperatura máxima foi superior à da temperatura mínima para os períodos de 1965 a 2010, em Araripina. Houve forte aumento da temperatura máxima e redução da temperatura mínima ao longo das décadas, com aumento de até $1,6 \circ C$ por década em junho e redução da temperatura mínima em até $0,45 \circ \mathrm{o}$ por década observada, em dezembro. A maior diferença entre as tendências para as duas variáveis (temp máx e min) ocorreu nos meses de abril e maio. Nos demais meses, a taxa manteve-se proporcionalmente semelhante (Figura 2).

\section{Araripina}

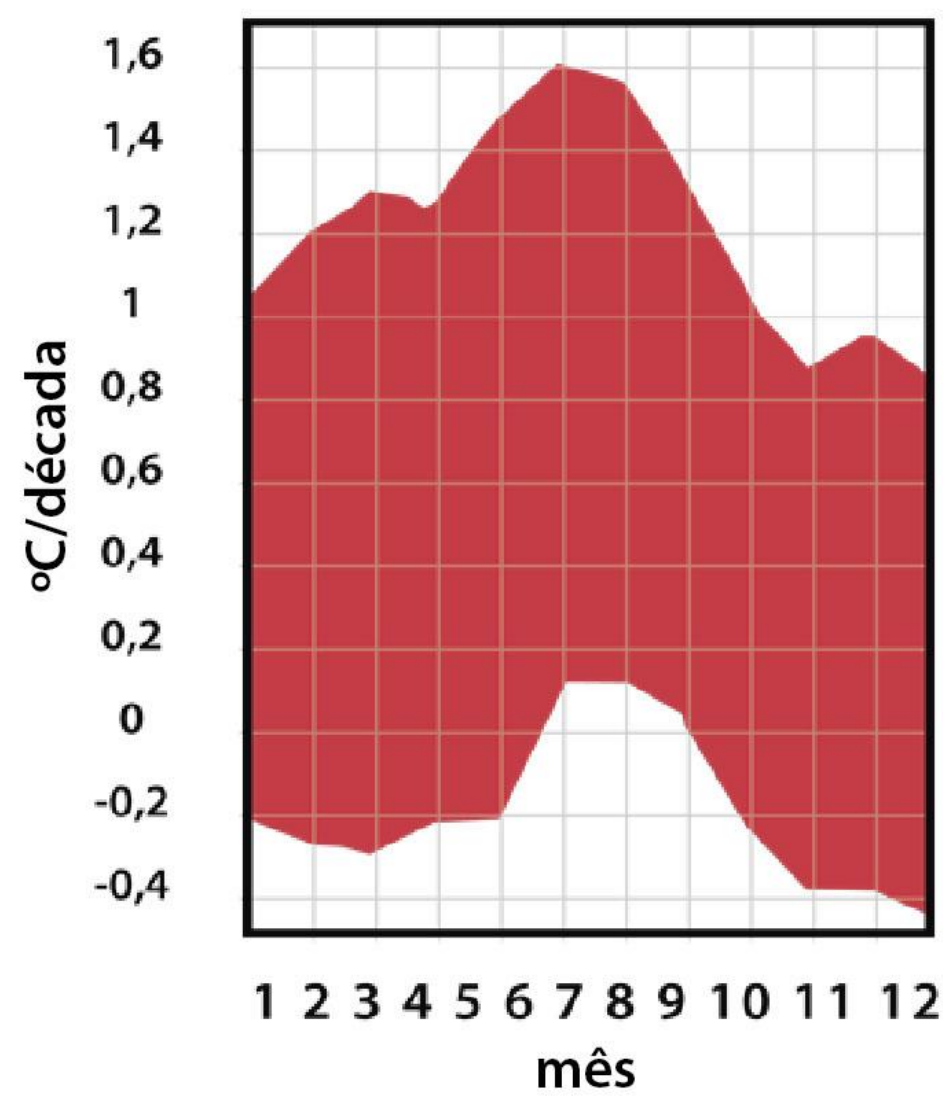

Figura 2: Taxas de variação decadal das temperaturas máximas e mínimas $\left({ }^{\circ} \mathrm{C} /\right.$ década) para cada mês do ano para Araripina.

Ao longo da série temporal analisada, 1965-2010, a evapotranspiração de janeiro a agosto, em Araripina, passou de $60 \mathrm{~mm}$ mês-1 a $120 \mathrm{~mm}$ mês-1, tal como ilustrado na Figura 3, valor semelhante ao observado nos meses secos, de outubro a dezembro.

Também se verificou que houve um aumento significativo das ETPs diárias ao longo de toda a série estudada e que mesmo nos meses mais frios os valores de ETP estão aumentando. O aumento progressivo dessas taxas em Araripina se mostra coerente com a elevação contínua das temperaturas nesta localidade.

Os resultados com as simulações e reanálises mostraram semelhanças entre os padrões de mudanças de temperatura ao longo da área em destaque (10S-5S, 43O-33O). Indicaram maior aquecimento no Oeste da região estudada, maior até do que aquele simulado em áreas próximas ao oceano. É importante ver a concordância entre o padrão de aquecimento diferencial da região litorânea com o da região interior do continente, com maior aquecimento constatado nas reanálises e capturado nas simulações com o modelo Eta, para o clima presente e futuro. O destaque vai para 
as simulações do clima para o futuro que prevê um aquecimento que é o dobro do valor simulado para o clima presente (Figura 4).

Em relação à simulação climática para o presente, o modelo regional não conseguiu capturar a taxa de aquecimento já detectada pela reanálise. Isso provavelmente se deve por causa de mudanças no uso da terra que estão indiretamente assimiladas nos dados da reanálise, mas não são representadas nas simulações com o modelo atmosférico regional. Já a diferença das taxas de aquecimento entre as simulações numéricas do modelo regional é devida às modificações nos campos das forçantes laterais provenientes do modelo global e das concentrações de $\mathrm{CO} 2$ atmosférico do modelo regional (mantendo a mesma vegetação em ambos os experimentos do clima presente e futuro).

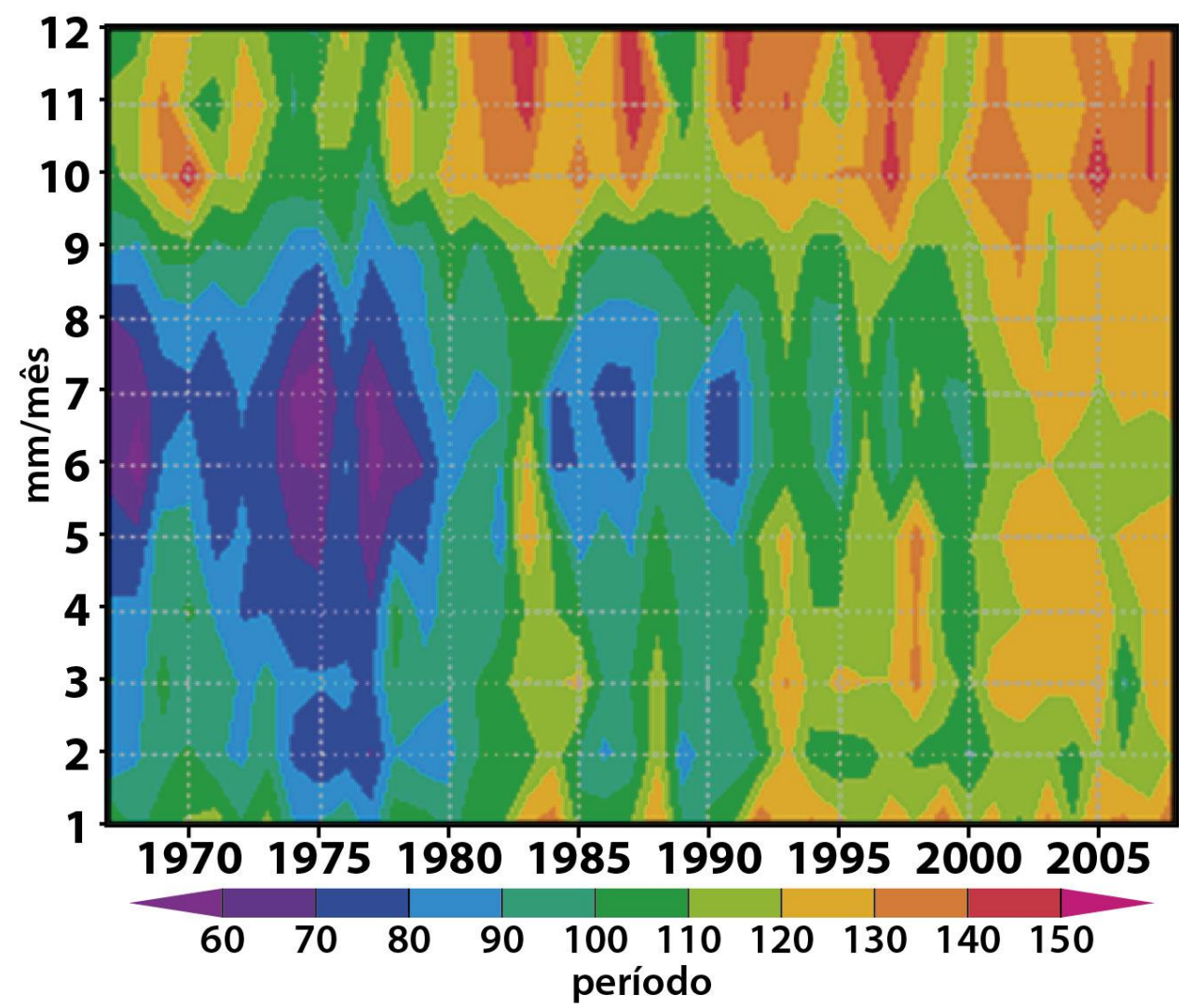

Figura 3: Evolução da evapotranspiração mensal entre 1965 e 2010, em Araripina (PE).

$\mathrm{Na}$ comparação entre o clima presente e a reanálise, constatou-se que os dados gerados com o modelo regional (Figura 4b) subestimaram a taxa de aquecimento já detectada sobre a área representada nos campos de reanálise (Figura 4a). A Figura 4a, mostra a variação da temperatura à superfície, como simulada pelas reanálises do NCEP, com taxa de variação de $2,065^{\circ} \mathrm{C} /$ século ou $0,207^{\circ} \mathrm{C} /$ década. A variação da temperatura à superfície sobre a área descrita acima, como simulado pelo modelo Eta, para o período de 1960 a 2000, mostra uma taxa de aquecimento médio de $0,082^{\circ} \mathrm{C} /$ década o que corresponde a aproximadamente $50 \%$ da variação nas temperaturas oriundas da reanálise para o mesmo período. A simulação com o Eta para o clima futuro (2010-2050) mostra uma tendência positiva de $0,196^{\circ} \mathrm{C} /$ década. Tal como para os perfis de temperatura, para 2010 2050, o modelo mostra um padrão semelhante com as simulações do passado (1960-2000), porém a magnitude prevista é o dobro. O cenário futuro para o lado ocidental do estado de Pernambuco produziu um valor de $0,11^{\circ} \mathrm{C} /$ década e um aquecimento futuro de $0,26^{\circ} \mathrm{C} /$ década. $\mathrm{O}$ aumento da concentração de $\mathrm{CO} 2$ de 380 para 760 possivelmente foi o fator determinante no crescimento da temperatura do ar nas simulações para o futuro em comparação aos dados de reanálises (Figura 4d). 
A análise de tendências para as temperaturas média do ar na superfície obtida com o modelo Eta-BESM para 1960-2000 (Figura 4c) mostra uma tendência positiva de $0,12^{\circ} \mathrm{C} /$ década no Sertão e $0,06^{\circ} \mathrm{C} /$ década para o restante do estado de Pernambuco. Estes valores de tendência foram resultados da integração do modelo com a concentração de $\mathrm{CO} 2$ atmosférico fixada em 374 ppm, representando as condições climáticas atuais e cobertura de vegetação inalterada.

(a) Reanalysis

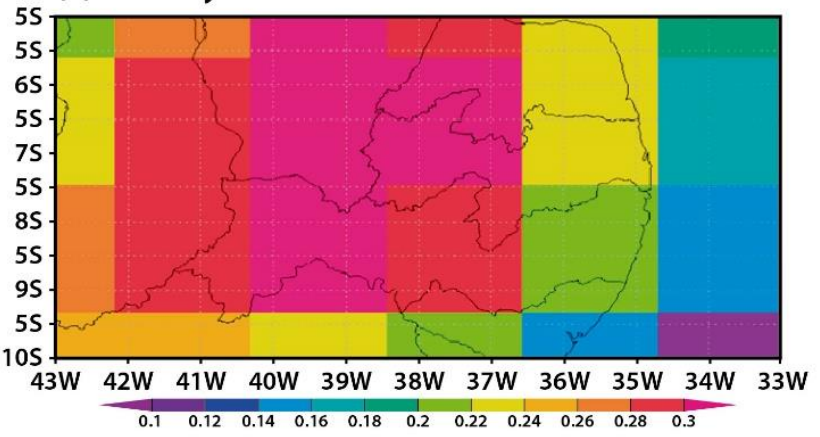

(c) Eta-BESM - past

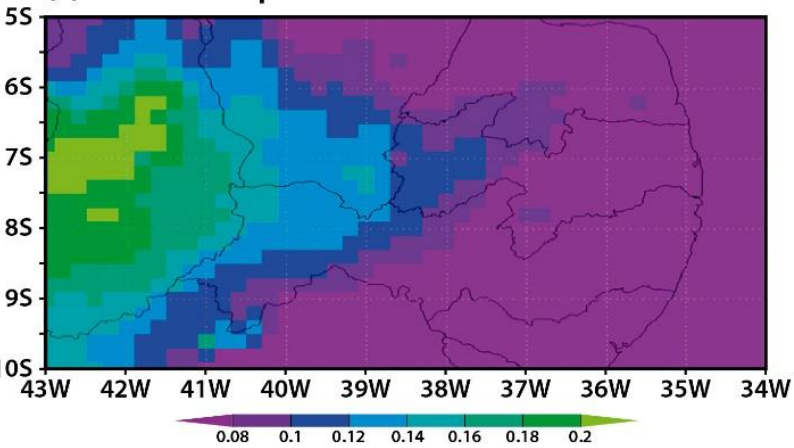

(b) Eta-HadCM3 - past

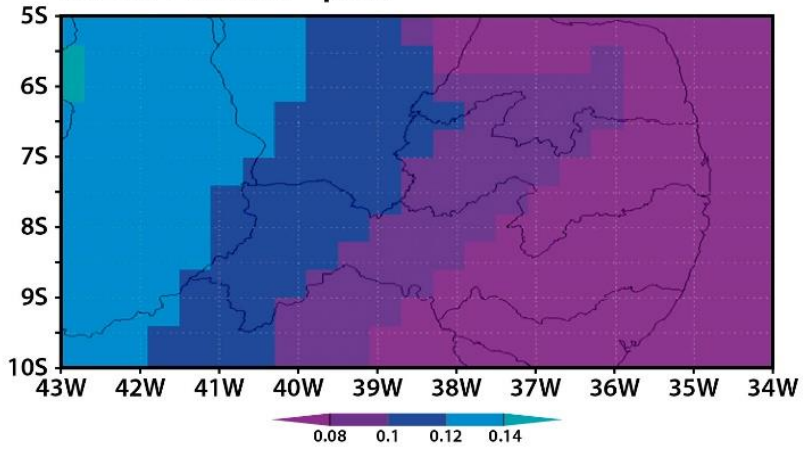

(d) Eta-HadCM3 - future

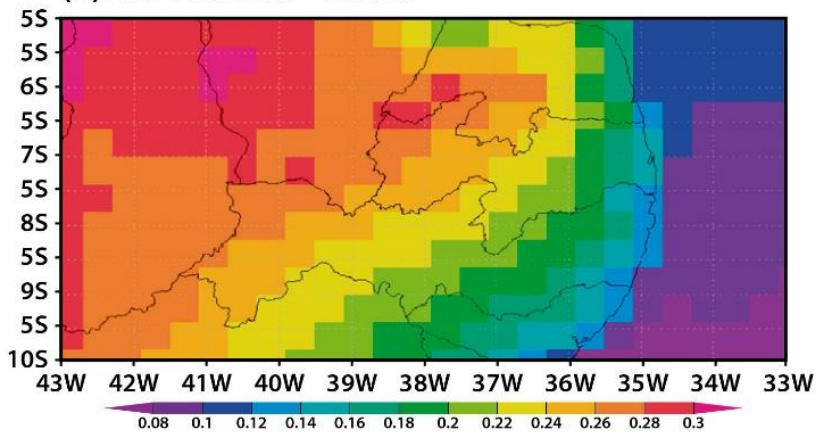

Figura 4: Tendências de temperatura média ( ${ }^{\circ} \mathrm{C} /$ década) simuladas pelo (a) NCEP-NCAR reanálises 1 , (b) Eta-HadCM3 e (c) modelos Eta-BESM de janeiro 1960 a dezembro 2000; e (d) Eta-HadCM3 cenário A2 de mudança climática para 2010-2050, para área de 10S-5S, 430-33O.

Os resultados das análises de tendências das temperaturas de superfície das simulações com o modelo regional Eta consideraram os valores médios na área de $13 \mathrm{~S}-5 \mathrm{~S}, 43 \mathrm{O}-33 \mathrm{O}$. Com essas saídas foi possível interpolar os dados diários futuros das temperaturas máximas e mínimas para Araripina. A variação da temperatura à superfície, simulada pelo modelo Eta, para o período de 2010 a 2050 e a taxa de aquecimento médio prevista para as máximas temperaturas máximas foi de 0,044oC/ano o que correspondeu a uma tendência de aumento de 1,76oC em 40 anos (Figura 5a). As variações futuras das mínimas das temperaturas mínimas mostraram uma taxa de aquecimento de 0,04oC/ano evidenciando um aumento de 1,60oC em 40 anos (Figura 5b).

\section{CONCLUSÕES}

O estudo sobre alterações climáticas para o semiárido pernambucano, utilizando séries temporais diárias de temperatura e de precipitação com mais de 40 anos, além de cenários futuros de mudanças do clima para o período de 2010 a 2050, evidenciou que os cenários regionalizados de mudanças climáticas para o futuro confirmaram as atuais tendências de aumento das temperaturas do ar para Araripina - semiárido pernambucano. Essas tendências indicaram sinais de processos de aridização, com aumento de até 1,6oC/década da temperatura máxima anual e diminuição da temperatura mínima em até $-0,4 \mathrm{oC} /$ década. 


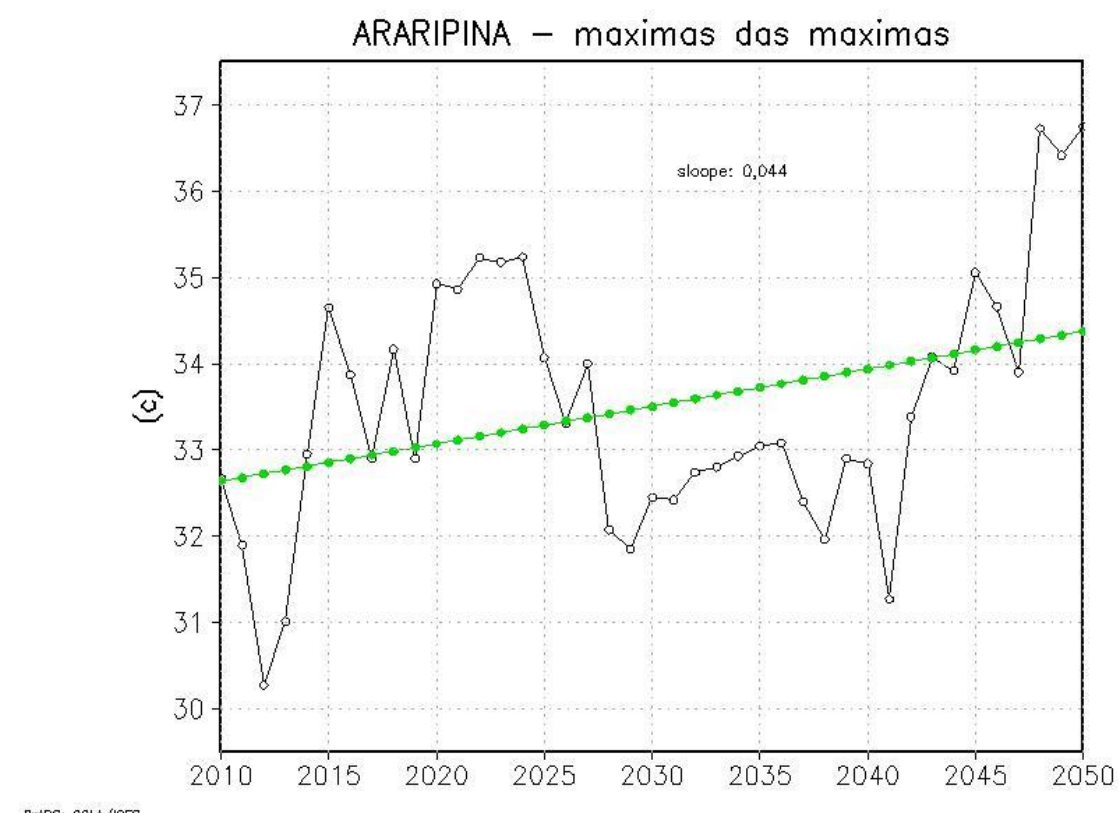

GrADS: COLA/IGES

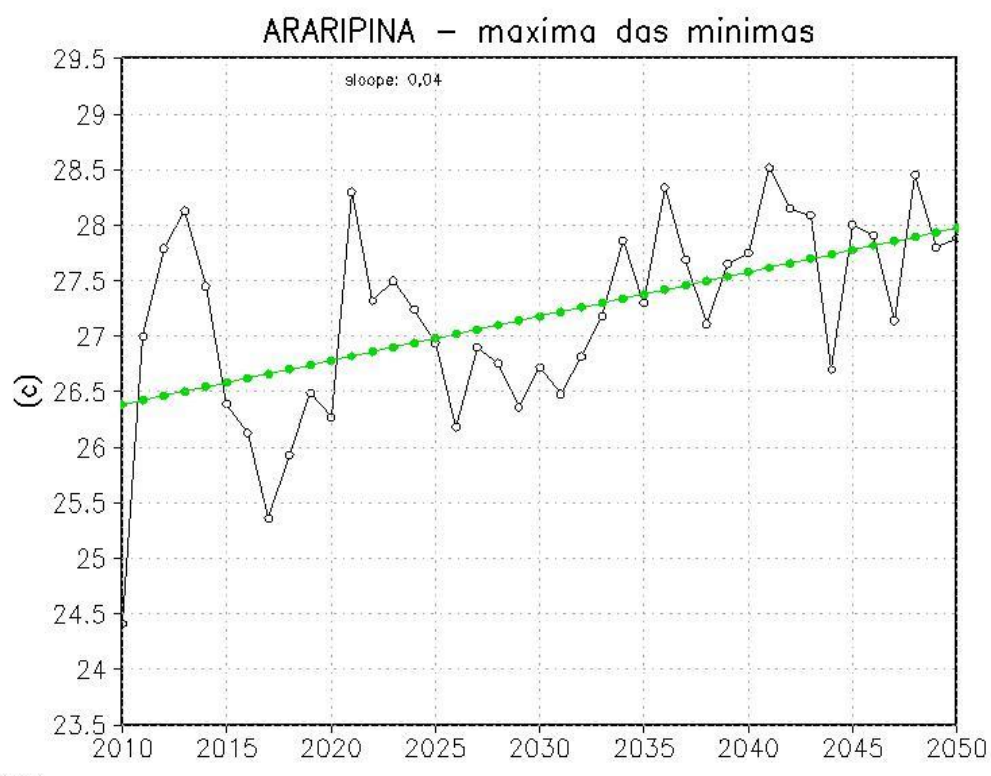

Figura 5 (a). Tendência das máximas temperaturas máximas, simulada com o modelo ETA/Lamepe para período de 2010 a $2050\left({ }^{\circ} \mathrm{C} /\right.$ década). (b). Tendência das temperaturas - máxima das mínimas, simulada com o modelo ETA/Lamepe para período de 2010 a $2050\left({ }^{\circ} \mathrm{C} /\right.$ década $)$

Nas análises de tendência das precipitações e pelos cálculos de balanço hídrico, se constatou a diminuição da precipitação total, diminuição da umidade do solo e aumento da evapotranspiração nas décadas de 1950-60 a 2010.

Outra importante constatação foi que, para o futuro, foi projetado um aquecimento de $1,960 \mathrm{C} /$ século e 0,26 oC/década, respectivamente, nas análises das tendências realizadas com os resultados das simulações climáticas, sobre grande parte do Nordeste do Brasil e Oeste de Pernambuco. Sendo o $\mathrm{CO} 2$ o fator com aumento contínuo na integração do modelo utilizado para simular o futuro, é plausível supor que tenha sido essa a causa do aumento das temperaturas nas simulações comparativas ao passado. 


\section{REFERÊNCIAS}

AMBRIZZI, T.; ROCHA, R. P.; MARENGO, J. A.; PISNITCHENKO, I.; ALVES, L. M. Cenários regionalizados de clima no Brasil para o Século XXI: Projeções de clima usando três modelos regionais. Relatório 3, Ministério do Meio Ambiente - MMA, Secretaria de Biodiversidade e Florestas -SBF, Diretoria de Conservação da Biodiversidade - DCBio Mudanças Climáticas Globais e Efeitos sobre a Biodiversidade- Sub projeto: Caracterização do clima atual e definição das alterações climáticas para o território brasileiro ao longo do Século XXI. Brasília, fevereiro, 2007

ASSAD, E. D.; PINTO, H. S. Aquecimento global e a nova geografia da produção agrícola no Brasil. EMBRAPA-CEPAGRI, São Paulo, agosto, 82 p, 2008.

CHOU, S. C, MARENGO, J. A, LYRA, A.; SUEIRO, G.; PESQUERO, J.; ALVES, L. M.; KAY, G.; BETTS, R.; CHAGAS, D.; GOMES, J. L.; BUSTAMANTE, J. F.; TAVARES, P. Downscaling of South America present climate driven by 4-member HadCM3 runs. Climate Dynamics. DOI 10.1007/s00382011-1002-8, 2011

CHOU, S. C; BUSTAMANTE J. F.; GOMES, J. Evaluation of ETA model seasonal precipitation forecast over South America. Nonlinear Process in Geophysics, v.12, p. 537-555, 2005.

HAYLOCK, M. R.; PETERSON, T. C.; ALVES, L. M.; AMBRIZZI, T.; ANUNCIAÇÃO, Y. M. T.; BAEZ, J.; BARROS, V. R.; BERLATO, M. A.; BIDEGAIN, M. CORONEL, I G.; CORRADI, V.; GARCIA, V. J.; GRIMM, A. M.; KAROLY, D.; MARENGO, J. A.; MARINO, M. B.; MONCUNILL, D. F.; NECHET, D.; QUINTANA, J.; REBELLO, E.; RUSTICUCCI, M.; SANTOS, J. L.; TREBEJO, I.; and VINCENT, A. Trends in total and extreme South American rainfall 1960-2000 and links with sea surface temperature. Journal of Climate, v. 19, 1490-1512. 2006

INTERGOVERNMENTAL PANEL ON CLIMATE CHANGE - IPCC. Climate Change - The physical science basis. Contribution of working group I to the fourth assessment report of the IPCC. Cambridge Univ. Press, Cambridge, 2007.

INTERGOVERNMENTAL PANEL ON CLIMATE CHANGE - IPCC. Summary for Policymakers. In: Climate Change 2013: The Physical Science Basis. Contribution of Working Group I to the Fifth Assessment Report of the Intergovernmental Panel on Climate Change Stocker, T. F., D. Qin, G.-K. Plattner, M. Tignor, S. K. Allen, J. Boschung, A. Nauels, Y. Xia, V. Bex and P. M. Midgley (eds.). Cambridge University Press, Cambridge, United Kingdom and New York, NY, USA. 2013.

LACERDA, F. F.; NOBRE, P.; SOBRAL, M. C.; LOPES, G. M. B.; CHAN, C. S. BRITO, E. Long term climate trends over Nordeste Brazil and Cape Verde. J Earth Sci Clim Change 2015, 6:8 http://dx.doi.org/10.4172/2157-7617.1000296

LACERDA, F. F.; SILVA JÚNIOR, H. D. DA, ASSAD, E. D., ASSIS, J. M. O., MOURA, M. S. B. Extremos e variabilidade climática no Nordeste brasileiro e em Pernambuco In: Mudanças Climáticas e Impactos Ambientais / organizador Josicleda Domiciano Galvíncio - Recife : Ed. Universitária da UFPE, 342 p. 1-23, 2010.

MARENGO, J. A. LINCOLN, M. ALVES, L. M.; VALVERDE, M. C.; LABORBE, R.; ROCHA, R. P. da. Eventos extremos em cenários regionalizados de clima no Brasil e América do Sul para o século XXI: Projeções de clima futuro usando três modelos regionais. Relatório 5, Ministério do Meio Ambiente MMA, Secretaria de Biodiversidade e Florestas - SBF, Diretoria de Conservação da Biodiversidade DCBio Mudanças Climáticas Globais e Efeitos sobre a Biodiversidade - Subprojeto: Caracterização do clima atual e definição das alterações climáticas para o território brasileiro ao longo do Século XXI. Brasília, fevereiro, 2007.

MCCABE, G. J.; MARKSTROM, S. L. A monthly water-balance model driven by a graphical user interface, p. 11, 2007.

NOAA/ National Weather Service National Centers for Environmental Prediction Climate Prediction Center http://www.esrl.noaa.gov/psd/data/gridded/data.ncep. reanalysis.html (acesso em 18mar2015)

NOBRE, C. A.; ASSAD, E. D. O. "Aquecimento Global e o Impacto na Amazônia e na Agricultura Brasileira" - INPE Eprint: Disponível em: http://www.sid.inpe.br/ePrint@80 /2005/09.12.12.51 v.1. 2005.

NOBRE, P. Mudanças Climáticas e desertificação: os desafios para o Estado Brasileiro. In: Desertificação e Mudanças Climáticas no Semiárido Brasileiro. Editores: R. C. C. Lima, A. M. B. Cavalcante e A. M. P. Marin, Instituto Nacional do Semiárido - INSA, pp 25-35, ISBN: 978-85-64265-02-8, 2011. 
NOBRE, P. SIQUEIRA, L. S. P.; ALMEIDA, R. A. F. de; MALAGUTTI, M.; GIAROLLA, E.; CASTELÃO, G. P.; BOTTINO, M. J.; KUBOTA, P.; FIGUEROA, S. N.; COSTA, M. C.; BAPTISTA JR. M.; IRBER JR. L.; MARCONDES, G. G. Climate simulation and change in the Brazilian Climate Model. J. Climate, 26, 6716-6732, DOI: 10.1175/JCLI-D-12-00580, 2013.

SANTOS, C. A.; BRITO, J. I. B. Análise dos índices de extremos para o semi-árido do Brasil e suas relações com TSM e IVDN. Revista Brasileira de Meteorologia, v. 22, n. 3, 303-312. 2007.

SILLMANN, J.; KHARIN, V. V.; ZWIERS, F. W.; ZHANG, X.; BRONAUGH, D. Climate extremes indices in the CMIP5 multi-model ensemble. Part 1: Model evaluation in the present climate. J. Geophys. Res., doi:10.1002/jgrd.50203. 2013a

SILLMANN, J.; KHARIN, V. V.; ZWIERS, F. W.; ZHANG, X.; BRONAUGH, D. Climate extremes indices in the CMIP5 multi-model ensemble. Part 2: Future projections. J. Geophys. Res., doi:10.1002/jgrd.50188. 2013b

SILVA, A. G; AZEVEDO, P., Índices de tendências de Mudanças Climáticas no Estado da Bahia. Engenheiria Ambiental, v. 5, 141-151. 2008.

TRENBERTH, K. E., JONES, P. D.; AMBENJE, P.; BOJARIU, R.; EASTERLING, D.; KLEIN TANK, A.; PARKER, D.; RAHIMZADEH, F.; RENWICK, J. A.; RUSTICUCCI, M.; SODEN, B.; and ZHAI, P. Observations: Surface and Atmospheric Climate Change. In: Climate Change 2007: The Physical Science Basis. Contribution of Working Group I to the Fourth Assessment Report of the Intergovernmental Panel on Climate Change [Solomon, S., D. Qin, M. Manning, Z. Chen, M. Marquis, K.B. Averyt, M. Tignor and H.L. Miller (eds.)]. Cambridge University Press, Cambridge, United Kingdom and New York, NY, USA. 2007.

ZHANG, X., HEGERL, G., ZWIERS, F., KENYON, J. Avoiding inhomogeneity in percentile-based indices of temperature extremes. Journal of Climate, v. 18, 1641-1651. 2005.

ZHANG, X; YANG, F. RClimDex (1.0) User Manual, 23 p, 2004. 